\title{
Future NASA Programs and Funding Support
}

\author{
Hashima Hasan \\ NASA Headquarters, Washington DC 20546, USA
}

\begin{abstract}
The future of Astronomy and Physics programs in the Office of Space Science is presented in the context of the overall NASA Strategic Plan. The highlights of the new road maps for the Origins and Structure and Evolution of the Universe science themes are given. A new initiative, Beyond Einstein, is discussed. New opportunities for technology development, building small missions, and conducting basic research and analysis to support NASA's strategic goals are outlined. The status of NASA's archives for data from its astronomy missions is presented.
\end{abstract}

\section{Introduction}

The science conducted by OSS is divided into four scientific themes.

- Astronomical Search for Origins (ASO)

- Structure and Evolution of the Universe (SEU)

- Sun Earth Connection (SEC)

- Solar System Exploration (SSE)

Road maps produced by the scientific community for each of these science themes form the basis for the Space Science Strategy. The first two science themes listed above, ASO and SEU, encompass the Astronomy and Physics program.

\section{Astronomy and Physics Program}

\section{Astronomical Search for Origins}

The science of the ASO science theme is focused around the following three research areas and the related science questions.

\section{Emergence of the Modern Universe}

-How did the cosmic web of matter organize into the first stars and planets?

-How do different galactic ecosystems (of stars and gas) form and which can lead to planets and living organisms?

Stars and Planets

-How do gas and dust become stars and planets?

-Are there planetary systems around other stars and how do their architectures and evolution compare with our own solar system?

Habitable Planets and Life

-What are the properties of giant planets orbiting other stars?

-How common are terrestrial planets? What are their properties? Which of them might be habitable?

-Is there life on planets outside the solar system?

The ASO road map defines a series of space flight missions, often supported by 
ground based programs which may be required as scientific or technology precursors to a flight program. The philosophy followed by both ASO and SEU is that the science and technology feeds forward from one mission to the next.

The ASO mission, Space Infrared Telescope Facility (SIRTF), the last of NASA's Great Observatories, is scheduled for launch in August 2003. The Infrared Astronomy (SOFIA), James Webb Space Telescope (JWST), Space Interferometry Mission (SIM), Terrestrial Planet Finder (TPF) and Kepler are in development. Ground based missions in support of SIM and TPF are the Keck interferometer, and the Large Binocular Telescope Initiative (LBTI). Vision missions, such as large UV and IR telescopes, leading ultimately to a Planet Imager (PI) and Life Finder (LF) are mentioned as future possibilities.

Structure and Evolution of the Univ aunch in August 2003. Flight missions in development are the Stratospheric Observatory for erse

The SEU road map is divided into two parts. The first, Beyond Einstein, describes a bold new initiative which aims to address some of the unsolved questions raised by Einstein's theories. The second, Cycles of Matter, addresses the remaining SEU science.

The three tantalizing questions Beyond Einstein attempts to answer are as follows.

- What powered the big bang?

- What happens at the edge of a black hole?

- What is dark energy?

The road map proposes three inter-linked elements that work together.

Einstein Great Observatories providing breakthrough increases in capabilities to address all Beyond Einstein science:

-LISA: Gravitational waves from merging black holes and the early Universe

-Constellation-X: Spectroscopy close to the event horizon of black holes and place constraints on dark side of the Universe

Einstein Probes to address focused science objectives:

-Determine the nature of the Dark Energy

-Search for the signature of inflation in the microwave background

- Take a census of Black Holes of all sizes in the local Universe

A technology program, theoretical studies and an education program to inspire future generations of scientists and engineers towards the vision:

-Directly detect the gravitational waves emitted during the Big Bang

-Image and resolve the event horizon of a Black Hole

\section{Explorer and Discovery Programs}

The objective of the Explorer and Discovery programs is to provide frequent flight opportunities for world-class scientific investigations from space utilizing innovative, streamlined, and efficient management approaches within ASO, SEU and SEC space science themes for Explorer and SSE and "search for planets" component of ASO for Discovery. The programs seeks to enhance public awareness of, and appreciation for, space science and to incorporate educational and public outreach activities as integral parts of space science investigations. 
The Explorer Program provides several classes of projects:

- Medium-class Explorers (MIDEX) - are investigations characterized by definition, development, launch service, and mission operations and data analysis costs not to exceed $\$ 180$ million (FY02 \$) total cost to NASA.

- Small Explorers (SMEX) - are investigations characterized by definition, development, launch service, and mission operations and data analysis not to exceed $\$ 120$ million (FY03 \$) total cost to NASA.

- University-class Explorers (UNEX) - are investigations characterized by definition, development, launch service, and mission operations and data analysis costs not to exceed $\$ 15.0 \mathrm{M}$ (FY00) total cost to NASA. (Currently on hold).

- Missions of Opportunity (MO) - are investigations characterized by being part of a non-NASA space mission of any size and having a total cost to NASA under $\$ 35$ million (FY03). They are conducted on a no-exchange-of-funds basis with the organization sponsoring the mission. NASA solicits proposals for Missions of Opportunity with each Announcement of Opportunity (AO) issued for UNEX, SMEX, and MIDEX investigations.

The cost cap for Discovery missions is $\$ 299$ million (FY01 \$), with an MO option as above.

\section{Technology Development Opportunities}

Several technology development opportunities exist for investigators working in U.S. institutions. Collaboration with non-U.S. investigators is permitted on a no exchange of funds basis. The following is a sample of existing technology development programs within OSS.

Focused Technology Programs

- The primary source of advanced space observatory technology

Space Science Research and Analysis Technology Programs

-Appropriate technology source for Explorers and PI class instruments

-Limited effectiveness in supporting strategic technology needs of ASO/SEU

Partnerships With Other Agencies, e.g.

-Advanced Mirror System Demonstrator (AMSD) Partnership

-Advanced Hybrid Mirror Demonstrator (AHM) with X-Sat

Small Business Innovation Research(SBIR)/Small Business Technology Transfer (STTR)

-Continues to be a viable source of niche technologies (Cryogenic high precision deformable mirrors, for example)

Aerospace Technology(AT) Programs

-Restructuring of AT programs looks promising for Science driven technology

New Millennium Program(NMP)

- has yet to fly an astrophysics focused validation mission

-ST7/DRS will be first

\section{Research Opportunities for Space Science (ROSS)}

Scientists working in U.S. institutions have the opportunity to perform basic research to support the goals of OSS by submitting proposals in response to 
the ROSS solicitation. All proposals undergo a competitive peer review process before being selected for funding. The Astronomy and Physics component of ROSS can be summarized as follows.

Astronomy 83 Physics Research and Analysis

- Suborbital (sounding rocket and balloon payloads)

- Supporting Technology

- Laboratory Astrophysics

- Fundamental Physics

- Ground Based Astronomy (U.S. Government funded institutions only) Astrophysics Theory Program

Long Term Space Astrophysics

Astrophysics Data Program

Origins of Planetary Systems (partially)

Astrobiology Instrument Development (partially)

\section{NASA's Astrophysics Data Archives and Data Services}

\section{Archives:}

-HEASARC (High Energy Astrophysics) at GSFC - holdings for RXTE, HETE, Chandra, etc.

-MAST (Multi-mission UV/Optical) at STScI - holdings are HST, GALEX, FUSE, etc.

-IRSA (Infra-red, submm) at Caltech - holdings are 2MASS, ISO, IRAS, soon SIRTF, etc.

-LAMBDA (Cosmic Microwave Background) at GSFC - holdings for WMAP, COBE, etc.

-MSC (Michelson Science - interferometry) at Caltech - holdings are for Keck interferometry data, etc.

Data services: -ADS (astrophysical Data Service) at SAO - holdings of scans of all journals and a great deal of astronomical literature.

-NED (NASA Extra-galactic Database) at Caltech - holdings of cross-correlated extra-galactic data in all wavelengths.

Acknowledgments. The contents of this paper are taken from a variety of NASA publications given in the reference section.

\section{References}

NASA's Vision http://www.gsfc.nasa.gov/nasavision/02_066.htm

NASA's Office of Space Science http://spacescience.nasa.gov/

Origins Road map http://origins.jpl.nasa.gov/library/roadmap03/

SEU Roadmap http://universe.nasa.gov/be/roadmap.html

Explorer Program http://explorers.gsfc.nasa.gov/

Discovery Program http://explorers.gsfc.nasa.gov/

OSS Research Opportunities http://research.hq.nasa.gov/code_s/code_s.cfm Astronomy and Astrophysics Data Archives http://nssdc.gsfc.nasa.gov/astro/ 